Overall, our results show that the immobilized VEGF increases the EC survival both in vitro and in vivo by downregulating miR-17, of which main gene targets are CDKN1A and ZNF652 to exert its function in the survival of the cells.

\section{A NOVEL SELECTIVE INHIBITIOR FOR PLASMA MEMBRANE CALCIUM ATPASE 4 IMPROVES VEGF- MEDIATED ANGIOGENESIS}

\begin{abstract}
${ }^{1}$ Sathishkumar Kurusamy*, ${ }^{2}$ Dolores Lopez-Maderuelo, ${ }^{3}$ Robert Little, 'David Cadagan, ${ }^{1}$ Clare Murcott, ${ }^{1}$ Rhiannon Baggott, ${ }^{3}$ Delvac Oceandy, ${ }^{1}$ Farjana Begam Rowther, ${ }^{1}$ Weiguang Wang, ${ }^{4}$ Ludwig Neyses, ${ }^{3}$ Elizabeth Cartwright, ${ }^{5}$ Juan Miguel Redondo, ${ }^{1}$ Angel luis Armesilla. 'University of Wolverhampton; ${ }^{2}$ Centro Nacional de Investigaciones Cardiovasculares; ${ }^{3}$ Institute of Cardiovascular Sciences, University of Manchester; ${ }^{4}$ Institute of Cardiovascular Sciences, University of Manchester, UK and University of Luxemburg, Luxemburg; ${ }^{5}$ Centro Nacional de Investigaciones Cardiovasculares; *Presenting Author
\end{abstract}

\subsection{6/heartjnl-2016-309890.199}

Cardiovascular diseases such as ischaemic heart disease, peripheral arterial disease and stroke are leading causes of death worldwide. Therapeutic angiogenesis, which can improve the formation of new blood vessels in the ischaemic organ, provides a valuable tool for treating cardiovascular diseases. Angiogenesis, a complex blood vessel formation process, involves the participation of several pro- and anti-angiogenic factors. Among them, pro-angiogenic factor Vascular Endothelial Growth Factor (VEGF) has been identified to play a critical role in pathological angiogenesis. Pre-clinical studies demonstrate that VEGF-based pro-angiogenic therapies result in successful reperfusion of the ischaemic organ in animal models. In our previous study, we reported a novel role for the Plasma Membrane Calcium ATPase 4 (PMCA4) as a negative regulator of angiogenic processes mediated by VEGF. Here, we hypothesised that selective inhibition of PMCA4 with the small molecule aurintricarboxylic acid (ATA) will improve VEGF-driven angiogenesis in vitro and an in vivo model of mouse limb ischaemia.

Consistent with this hypothesis, we demonstrate in this work that inhibition of PMCA4 by treatment with ATA significantly increases the activity of calcineurin/NFAT pathway and the subsequent expression of the NFAT-dependent, pro-angiogenic protein RCAN1.4 in VEGF-stimulated endothelial cells. Additionally, ATA treatment reduces the level of membraneassociated calcineurin, suggesting that enhancement of calcineurin signalling is the result of a disruption of the interaction between PMCA and calcineurin. Moreover, ATA treatment strongly enhances endothelial cell motility and capillary-like formation in matrigel assay. Furthermore, ATA significantly enhances MLEC motility in PMCA4 $+/+$ (wild type), but not in PMCA4 -/- (knock out) cells, conforming that ATA-mediated inhibition of PMCA4 is implicated in the increase of migration exerted by ATA. Importantly, long-term exposure of endothelial cells to ATA has no changes in cell viability, highlighting the potential clinical application of ATA. In this sense, Post-ischaemic reperfusion of ischaemic limbs in animals treated with ATA is significantly higher than in control-treated animals. The data from this study indicated that modulation of the activity of PMCA4 by treatment with ATA might have important clinical applications to promote blood vessel formation in human diseases associated with insufficient angiogenesis.

\section{0 DEDIFFERENTIATED OR REBORN AGAIN? ELUCIDATING THE CHROMATIN REMODELLING MECHANISMS DURING ENDOTHELIAL CELL REPROGRAMMING FOR CARDIOVASCULAR THERAPY}

Rachel Caines*, Sophia Kelaini, David Grieve, Alan Stitt, Andriana Margariti. Queen's University Belfast; *Presenting Author

10.1136/heartjnl-2016-309890.200

The vascular endothelium is central to cardiovascular homeostasis. Repair and regeneration of endothelial cells (ECs) has been an important research focus for a number of years. The recent ability to derive ECs through cell reprogramming has opened new avenues. Reprogramming somatic cells to ECs is in its infancy, but the road ahead looks very promising.

A new reprogramming strategy has ruled out safety issues concerned with teratoma formation. Cells exposed to reprogramming factors for 4 days become epigenetically primed and have been defined as Partially induced Pluripotent Stem (PiPS) cells. They do not transverse pluripotency, and so do not form tumours. They have shown the ability to be differentiated into ECs by culture conditions. Efficiency of reprogramming has increased from $0.01 \%$ to $30-40 \%$ in the case of PiPS-ECs, but there is scope for improvement as the underlying mechanisms are still unclear. The role of epigenetics in reprogramming has come to the forefront recently and the ability to generate a homogenous and functional EC population will be best sought through chromatin remodelling mechanisms.

A protein found to be crucial in 4-day reprogramming was SETSIP, or SET similar protein. SETSIP has high sequence homology to SET with an additional 10 amino acids at the $\mathrm{N}$ terminus. SET plays roles in chromatin remodelling as a transcriptional regulator and roles in differentiation, apoptosis and cell cycle progression. The study aims to elucidate a robust and efficient protocol for the production of a homogenous and functional EC population for use in personalised cardiovascular medicine.

SETSIP has been overexpressed and knocked out of early PiPS-ECs and iPS-ECs to observe the effect on EC reprogramming. Luciferase assays have been undertaken to understand the EC specific pathways regulated by SETSIP. Experiments have also been performed to establish the effect of treatment with VEGF on SETSIP expression and EC differentiation. Future work will involve the employment of CRISPR technology to create a SETSIP deficient cell line to observe the differentiation potential of the cells and phenotype of any derived ECs.

SETSIP was found to translocate to the cell nuclei, and capable of regulating expression of important EC markers. The functional consequences of this were assessed in vitro and in vivo where SETSIP was found to be important for the formation of vascular tubules. Furthermore, epigenetic modulators such as $\mathrm{CBP} / \mathrm{p} 300$ were identified as potential mediators of the gene regulatory effects of SETSIP in ECs. These results represent an important step forward in understanding the process of EC reprogramming for use in regenerative medicine.

These findings provide knowledge of the intricate processes during EC reprogramming not only to support the scientific validity of the newly generated ECs but also to ensure the safety of bringing cellular reprogramming to the bedside of cardiovascular patients. 